Revista de Investigación Educativa 25

julio-diciembre, 2017 | ISSN 1870-5308 | Xalapa, Veracruz

Instituto de Investigaciones en Educación | Universidad Veracruzana

\title{
La formación de profesionales en desarrollo sustentable en un programa de educación superior intercultural
}

\section{The training of professionals in sustainable development in an intercultural higher education program}

\author{
Dr. Joaquín Peña Piña \\ Profesor-Investigador \\ Universidad Intercultural de Chiapas, México \\ joaquinpp@unich.edu.mx
}

El modelo educativo intercultural a nivel superior tiene un amplio potencial en la formación integral de jóvenes profesionales de acuerdo con su contexto. Sin embargo, como todo modelo educativo, su instrumentación depende de la participación conjunta de estudiantes, profesores, directivos y, en este caso, de los actores comunitarios. La formación profesional se da en el marco del intercambio y diálogo de saberes que contribuya a la construcción desde el mismo estudiante hacia la sustentabilidad y la interculturalidad. El objetivo es compartir la experiencia formativa en el programa académico de desarrollo sustentable de la Universidad Intercultural de Chiapas que resulta, a partir de las evidencias generadas en las primeras generaciones, en un modelo de fortalecimiento del diálogo de saberes. Se concluye que este modelo es acorde a las necesidades formativas de las actuales y futuras generaciones.

Palabras clave: Formación universitaria; perspectiva intercultural; desarrollo sustentable; Universidad Intercultural de Chiapas.

The intercultural educational model at the higher level has a great potential in the integral formation of young professionals according to their context. However, like any educational model, its implementation depends on the joint participation of students, teachers, managers and, in this case, community actors. Professional training takes place within the framework of the exchange and dialogue of knowledge that contributes to the construction of the same student to sustainability and interculturality. The objective is to share the training experience in the academic program of sustainable development at the Universidad Intercultural de Chiapas, which results, based on the evidence generated in the first generations, in a model of strengthening the dialogue of knowledge. It is concluded that this model is in accordance with the training needs of current and future generations.

Key words: University education; intercultural perspective; sustainable development; Universidad Intercultural de Chiapas. 


\title{
La formación de profesionales en desarrollo sustentable en un programa de educación superior intercultural
}

\author{
The training of professionals in sustainable development \\ in an intercultural higher education program
}

\section{Introducción}

\begin{abstract}
más de una década de la instrumentación del modelo educativo en las universi$A_{\text {dades interculturales, destaca la experiencia en la Universidad Intercultural de }}$ Chiapas (UNICH), ubicada en el estado epónimo, en el sureste mexicano donde prevalece un fuerte rezago en la educación superior, aunque, a su vez, es una región que cuenta con una rica diversidad biocultural. Se trata de una propuesta académica con proyección al fortalecimiento de los procesos de desarrollo y la cultura con un enfoque intercultural, para contribuir a la formación de profesionales jóvenes e indígenas de las zonas rurales del país, cuyo acceso a la educación superior se ha visto limitado. En el caso de la UNICH, el proyecto educativo se inició en 2005, como parte de un sistema de universidades interculturales guiado por el modelo educativo de educación superior intercultural (Casillas \& Santini, 2006).
\end{abstract}

\section{Análisis de la experiencia educativa en la UNICH}

En esta experiencia se profundiza en la aplicación del modelo educativo en la Licenciatura en Desarrollo Sustentable (LDS) de la UNICH, cuya instrumentación se guía por el diálogo de saberes ${ }^{1}$ entre la universidad y la sociedad, a través de las comunida-

1. El diálogo de saberes se concibe como el proceso de interacción de los saberes y prácticas entre las culturas, especialmente importante en la crisis ambiental que, de acuerdo con Leff (2004, p. 1), es el síntoma del límite de la racionalidad fundada en una creencia insustentable, donde la universalidad y objetividad del conocimiento condujerona la economización y cosificación del mundo. En nuestra práctica profesional se considera ese diálogo tomando en cuenta a los saberes propios del estudiante, los saberes académicos del aula y los saberes locales de las comunidades de vinculación. Esta tríada de saberes genera una dinámica propia que impulsa un proceso de aprendizaje creciente y continuo a lo largo en la formación profesional, que permite avanzar en la comprensión y práctica de la sustentabilidad y la interculturalidad (UNICH, 2005). 
des de vinculación (Freire, 2007; Pérez \& Argueta, 2011). Específicamente, en la LDS se promueve la sustentabilidad ${ }^{2}$ como un planteamiento teórico en la búsqueda de un equilibrio en el desarrollo económico, social y ambiental, propuesto por la Comisión Mundial de Medio Ambiente y Desarrollo, cuya meta se dirige hacia un futuro más sustentable para las siguientes generaciones (Brundtland, 1987).

El perfil profesional del programa académico intercultural de desarrollo sustentable de la UNICH busca entre sus egresados atender las problemáticas, potencialidades y alternativas de las comunidades, tomando como eje formativo al diálogo de saberes para contribuir al desarrollo de propuestas acordes al contexto específico, al uso sustentable de los recursos naturales, al fortalecimiento de la cultura tradicional, el análisis y grado de estabilidad en el tejido social, y a la promoción de la gestión y autogestión comunitaria (UNICH, 2005).

Si bien el desarrollo sustentable y la educación intercultural representan paradigmas en construcción, la estrategia pedagógica está centrada en el diálogo de saberes y opera fundamentalmente en el eje de la vinculación comunitaria, desde un enfoque comunicativo que permite escuchar y reconocer al otro, especialmente para recuperar ese diálogo que se encuentra en la rica diversidad étnica de México (Navarrete, 2008). Un diálogo que por lo general está invisibilizado por los procesos de modernización y la educación moderna. Por ello, el eje de vinculación comunitaria que caracteriza al modelo educativo intercultural ${ }^{3}$ representa un espacio de oportunidad para promover un diálogo incluyente y participativo en una sociedad diversa y en una realidad donde conviven e interactúan las sociedades tradicional y moderna. La mayoría de las comunidades de vinculación donde trabajan los estudiantes son indígenas, por lo cual es necesario considerar algunos elementos para el trabajo comunitario (Pérez, 2005, p. 88). Entre ellos están la tierra, que confiere derechos y obligaciones sociales, el poder comunal, a través de las asambleas, el trabajo comunitario y el disfrute de la fiesta, donde se fortalece y se reconstruye la cultura y la identidad comunitaria. Dichos elementos tradicionales contrastan con las prácticas del mundo contemporáneo,

2. La polémica en los conceptos de desarrollo sustentable y desarrollo sostenible se ha mantenido por varios años, y con frecuencia se utilizan de manera indistinta. En la UNICH consideramos a la sustentabilidad como un concepto más pertinente en la enseñanza por su carácter dinámico y de transformación continua hacia la búsqueda de un equilibrio en la sociedad; por su parte, la sostenibilidad, desde nuestra experiencia, implica mantener una situación en el tiempo, poco probable en el desarrollo, con un carácter menos dinámico. Una discusión hacia la sustentabilidad ambiental que profundiza en este aspecto se encuentra en Gómez (2014).

3. La vinculación comunitaria es un eje transversal del modelo educativo intercultural, donde se establece la relación entre la universidad y la sociedad. Implica relaciones, compromiso y trabajo solidario mediado por el diálogo. 
ocasionando definiciones muy diversas sobre el proceso de vinculación comunitaria. Un equipo de estudiantes la define así: "es el espacio donde se establece la relación entre los estudiantes y la comunidad para intercambiar conocimientos, experiencias, prácticas y la generación de nuevos conocimientos, que contribuye a fortalecer la ideología de la comunidad y la de nosotros mismos" (Equipo de estudiantes en la comunidad Carmen Yalchuch, Huixtán, Chiapas, 2017).

El objetivo de esta experiencia educativa es analizar las características y la experiencia formativa en el desarrollo sustentable y la dinámica en la construcción del diálogo de saberes como parte de la vinculación comunitaria, que resulta en la propuesta de un modelo de fortalecimiento para su formación profesional. El análisis se realiza a partir de la experiencia directa con profesores y estudiantes en el programa académico en desarrollo sustentable de la UNICH (versiones 2005 y 2008), mediante el trabajo realizado en el aula y en las comunidades de vinculación.

\subsection{La vinculación comunitaria en el programa académico en desarrollo sustentable}

Como preámbulo se puede mencionar que en el cumplimiento de la misión y el logro educativo en la educación superior, las universidades cumplen con las funciones sustantivas de docencia, investigación y extensión, para promover procesos dinámicos y graduales de formación académica. En el caso de las universidades interculturales, se incorporan adicionalmente al proceso la enseñanza de las lenguas originarias y la vinculación comunitaria, que constituyen el sello característico del modelo educativo intercultural.

En resumen, la oferta educativa de la UNICH experimentó en una década un proceso de construcción y cambio en sus programas educativos, que inició con cuatro programas académicos dirigidos a fortalecer la cultura (Lengua y cultura, Comunicación intercultural) y los procesos de desarrollo local (Turismo alternativo y Desarrollo sustentable), que operaron y se fortalecieron desde 2005, obteniendo su acreditación en 2011 por los Comités Interinstitucionales de Evaluación de la Educación Superior (CIEES). La oferta educativa de la UNICH se amplió en 2012 con los programas de Derecho intercultural y Medicina con enfoque intercultural, y su cobertura se extendió de la sede San Cristóbal de Las Casas hacia otras regiones de Chiapas con presencia indígena (Yajalón, Oxchuc, Las Margaritas y Salto de Agua).

El modelo educativo intercultural en desarrollo sustentable cuenta con el eje transversal de vinculación comunitaria como articulador de los otros ejes académi- 
cos formativos (Tabla 1). Así, el eje de vinculación integra a las asignaturas de cada semestre de manera vertical y horizontal, a través de un proceso educativo de complejidad creciente, que contribuye a la formación integral del estudiante mediante la interacción de los saberes disciplinarios y comunitarios.

En el caso del programa 2005, los ejes formativos que alimentan al eje de vinculación se identificaron siguiendo la denominación convencional de los tres ámbitos teóricos del desarrollo sustentable (político-económico, social-cultural, ecológicoambiental), aplicados con un enfoque cognitivo guiado por la taxonomía de Bloom a partir del tercer semestre (conocer, comprender, aplicar, analizar, sintetizar, evaluar), promoviendo un aprendizaje gradual, multidisciplinario e integral en la formación educativa del estudiante. Posteriormente, en el programa 2008 los ejes formativos se denominaron de acuerdo con los principios del modelo educativo intercultural (disciplinar y sociocultural), guiados por un enfoque de autogestión como elemento esencial en la sustentabilidad y el desarrollo local (diálogo de saberes, trabajo comunitario, estrategias locales, gestión y mediación, evaluación). A pesar de sus diferencias en la vinculación comunitaria y en el enfoque cognitivo y de autogestión de cada programa (Tabla 1), sus alcances no fueron excluyentes sino complementarios y enriquecieron su perspectiva. El logro educativo entre los estudiantes se manifestó en el entendimiento del proceso de desarrollo, en aspectos como la identificación de los problemas y necesidades de la comunidad, la valoración del trabajo colectivo, el interés por los problemas sociales, los procesos de acompañamiento y fortalecimiento comunitario, las capacidades de gestión y autogestión, así como un proceso continuo y necesario de reflexión y diálogo de saberes desde una visión multidisciplinaria y multidimensional. ${ }^{4}$

4. La visión multidisciplinaria es fundamental para el estudio del desarrollo ante la necesidad de entender dicho proceso desde diversas disciplinas y así entender la realidad misma en su complejidad y diversidad. Todo ello en contraste con los programas académicos disciplinares, como los de biología, agronomía, antropología entre otros, donde sólo se profundiza en una única visión. En la medida en que las diversas disciplinas establecen relaciones definidas y articuladas se estaría avanzando en la interdisciplinariedad. La visión multidimensional hace referencia al objeto de estudio o la realidad desde diversas dimensiones de análisis como la dimensión individual, familiar y comunitaria, que puede extenderse a nivel a la dimensión regional, nacional y global, de acuerdo con las necesidades de estudio y análisis. 


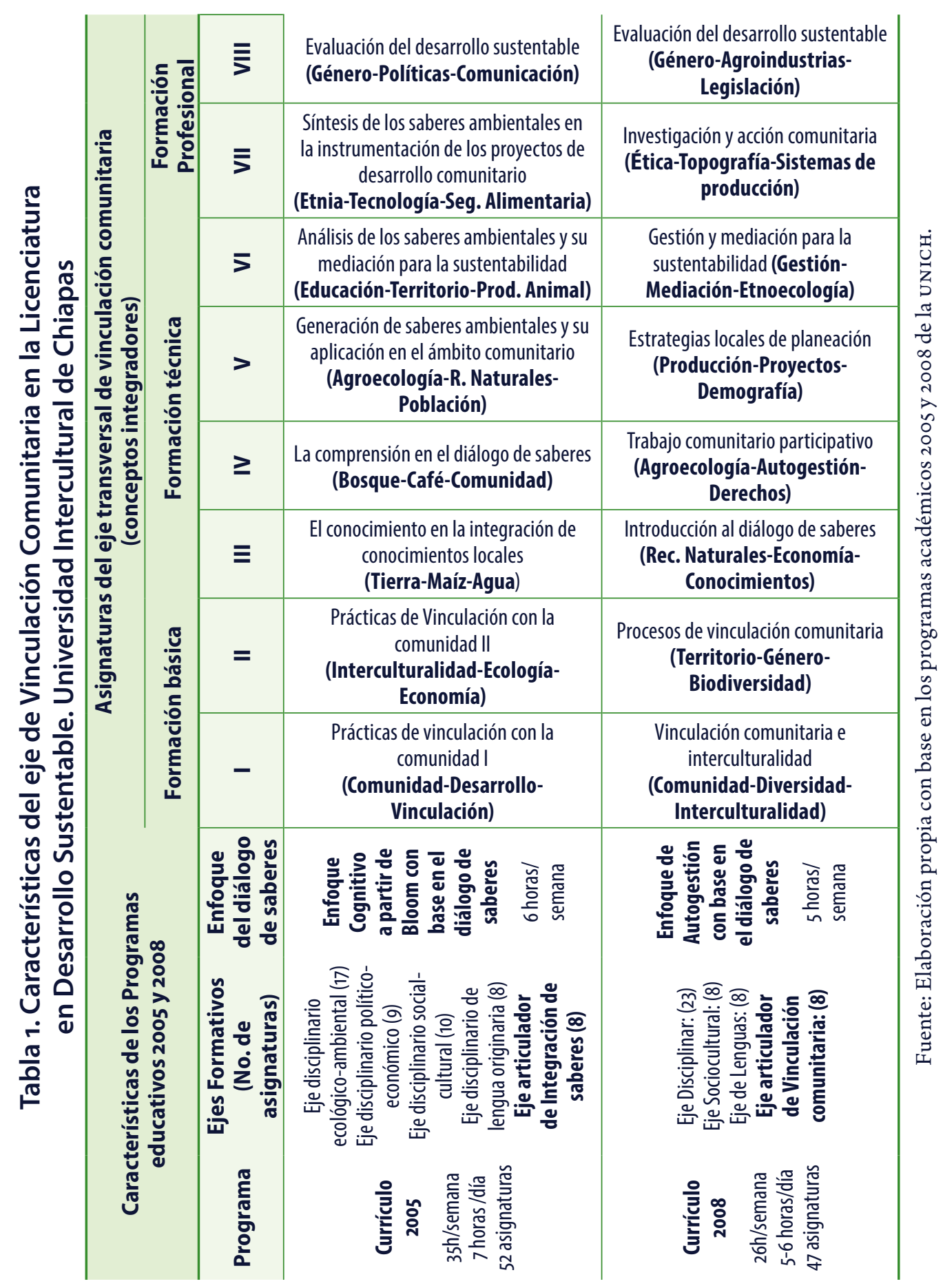




\subsection{Las contribuciones de la vinculación comunitaria como eje formativo}

En este análisis, el eje de vinculación comunitaria es el espacio que retoma todo tipo de saberes y prácticas a través del diálogo de saberes y como promotor de la interculturalidad en el campo educativo-formativo. Aquí es donde los estudiantes pueden reflexionar y entender cómo los actores locales conciben su desarrollo y cultura local, para proponer las alternativas de solución más adecuadas a su contexto. Esto significa que en el proceso de vinculación comunitaria el estudiante va adquiriendo un compromiso social a la par de su propia formación profesional, generando el interés en los múltiples aspectos de la vida comunitaria, de los conocimientos y las prácticas, y en el marco de un proceso de acompañamiento responsable y solidario. Un ejemplo de lo que representa el diálogo de saberes, su comprensión y práctica entre los estudiantes se expresa así: "el diálogo de saberes es un aprendizaje mutuo que busca promover la construcción social del conocimiento mediante el intercambio de ideas, creencias, nociones, prácticas, historias, vivencias y emociones, para alcanzar la comprensión y la plenitud de la vida" (equipo de estudiantes de vinculación comunitaria del $8^{\circ}$ semestre en la comunidad de Xulumó, municipio de Aldama, Chiapas, 2016). Lo que demuestra que el diálogo de saberes es un concepto amplio que va más allá de un intercambio de ideas o palabras.

Mediante este proceso de vinculación comunitaria, se contribuye al reconocimiento de la educación no formal de la comunidad y al fortalecimiento de la educación formal universitaria, es decir, la confluencia de la educación organizada y sistematizada en las aulas con la educación que la población posee y genera en sus comunidades. Así, mediante la vinculación comunitaria el estudiante fortalece sus capacidades de investigación y desarrolla habilidades para establecer relaciones sociales con diversos actores, identifica conocimientos, valores y actitudes locales, y adquiere diversas destrezas para la acción comunitaria al poner en práctica los recursos metodológicos y teóricos disponibles a partir de su formación.

La mayoría de estos principios son comunes a otras licenciaturas, pero en el caso específico de Desarrollo sustentable, acoge de manera amplia los conocimientos y prácticas tradicionales de las comunidades y las contrasta con las contemporáneas, para entender y emprender acciones integrales de desarrollo tomando en cuenta la cultura y el contexto. Al respecto, Walsh (2002, p. 135) considera que la interculturalidad, además de su connotación contra-hegemónica y de transformación de las 
relaciones sociales, también está relacionada con las diversas formas de producir y aplicar el conocimiento (epistémica), enfocada a revertir la designación de algunos conocimientos como legítimos y universales.

El impacto del proceso de vinculación comunitaria se identifica en la formación profesional de los estudiantes que adquieren experiencia en el mismo campo donde van a trabajar como egresados, en un espacio donde los estudiantes aprenden de la comunidad y la población aprende de ellos, desde un enfoque local y territorial, con la posibilidad de que el proceso educativo promueva una práctica transformadora de la realidad con los actores sociales.

La vinculación comunitaria se concibe, entonces, como un proceso de diálogo y acompañamiento a partir de la formación estudiantil en el aula y en la comunidad. Sin embargo, durante este proceso pueden existir barreras de diversa naturaleza que interfieren en su formación profesional. Entre las barreras más comunes mencionadas por los mismos estudiantes está el "paternalismo", que implica una dependencia de los apoyos del gobierno, la influencia y división ocasionada por los partidos políticos, los liderazgos locales y/o cacicazgos, la baja en el nivel de participación comunitaria o el desconocimiento de la lengua y las tradiciones, entre otras. Por su parte, los estudiantes en ocasiones no desarrollan un buen trabajo en equipo, no planean adecuadamente sus visitas a la comunidad, trabajan con pocas familias y no desarrollan habilidades para convocar y promover la participación comunitaria.

La formación del estudiante en desarrollo sustentable pretende ser integral y multidisciplinaria, lo que implica que el trabajo no puede estar centrado únicamente en el aula, sino en estrategias educativas adicionales que, en el caso de la LDS de la UNICH, logró conformar un modelo de fortalecimiento integral para el logro educativo profesional.

\section{Propuesta de un modelo educativo para el fortalecimiento del diálogo de saberes en el desarrollo sustentable}

Con base en la experiencia generada en los programas educativos 2005 y 2008 se identifican las siguientes acciones dentro del modelo educativo intercultural de la UNICH para el fortalecimiento del diálogo de saberes en el desarrollo sustentable. 


\subsection{La docencia y la pedagogía intercultural}

La docencia y la práctica docente con una pedagogía intercultural son aspectos centrales en el modelo educativo intercultural para la promoción del diálogo de saberes. En esta práctica se requiere que el profesor posea una visión pedagógica constructivista y se desempeñe como un "facilitador" del proceso educativo, donde los estudiantes logren ser los responsables de su propio proceso de aprendizaje. Asimismo, se precisa de una planta de profesores con experiencia profesional en proyectos de investigación y trabajo en comunidades, y una sólida formación profesional de acuerdo con su especialidad que combine la teoría y la práctica. En lo metodológico, son de gran utilidad la aplicación de la Investigación-Acción-Participativa (IAP) y las técnicas de discusión grupal como la Comunidad de Diálogo (Echeverría, 2016), que en conjunto han favorecido la participación activa, la discusión, el intercambio de ideas y el desarrollo de una visión crítica de los estudiantes.

\subsection{Incorporación de los conceptos integradores para el diálogo de saberes}

Los conceptos integradores (Tablas 1 y 2), representan conceptos teóricos que son clave para la formación académica de los estudiantes. Se pueden establecer hasta tres conceptos en cada semestre para articular el trabajo de las asignaturas disciplinares en un documento donde se integran interdisciplinarmente. Como ejemplo, en el primer semestre se trabaja sobre los conceptos de Comunidad-Desarrollo-Vinculación y las relaciones entre sí, esenciales para iniciar su formación, y así continua en los siguientes semestres. Estos conceptos constituyen una armazón teórica, evitan que los aportes disciplinarios de cada asignatura queden aislados y hacen posible que puedan confluir y articularse en la formación interdisciplinar.

\subsection{La vinculación en las comunidades con equipos de trabajo}

La vinculación comunitaria la realizan los estudiantes organizados en equipos de trabajo bajo su propia iniciativa, para darle seguimiento a una comunidad en todo el proceso de formación profesional (4 años). Se trata de que los estudiantes se mantengan en un mismo equipo de trabajo y con la misma comunidad, para acompañar un proceso de formación en tres etapas (Tabla 1): Una etapa básica se realiza en los primeros dos semestres, donde se sientan las bases del proceso de vinculación de mane- 
ra conceptual y metodológica. Posteriormente, una etapa técnica, donde se da inicio formal al proceso de vinculación comunitaria y, finalmente, una etapa de resultados y evaluación donde se afinan las estrategias de acompañamiento, se consolidan los beneficios del trabajo en equipo y se evalúa el proceso para el fortalecimiento del diálogo de saberes.

\subsection{Foros temáticos de análisis y discusión}

La organización de foros de análisis y discusión grupal tuvieron la finalidad de promover un debate de ideas sobre diversos temas específicos derivados de los ejes integradores de cada semestre para su análisis, discusión y reflexión. En esta experiencia se organizaron foros mediante un panel de expositores integrado por profesores, campesinos, estudiantes o especialistas centrados en un tema o eje. Durante el foro se inicia con una exposición de ideas del panel, como un insumo a discutir posteriormente en pequeños grupos que sesionan por separado, donde se enriquece con otros elementos e ideas de los participantes, la cual se registra en una relatoría. Los resultados de cada equipo se exponen en plenaria, y se cierra el evento con las conclusiones pertinentes. Estos foros contribuyen al fortalecimiento de las habilidades de discusión, análisis y reflexión propias con las de los actores externos.

\subsection{Los viajes de estudio grupales}

La organización de los viajes de estudio se planea con base en los conceptos integradores, para que los estudiantes conozcan experiencias profesionales desde los ámbitos socioeconómicos, productivos, culturales y ambientales en el estado de Chiapas. En ellos se combina la visita a los diversos ecosistemas, grupos étnicos y sistemas de producción (Tabla 2). Esto permite el reconocimiento de su propio estado en los diversos puntos geográficos de cada región, donde interactúan con los actores sociales para conocer sus saberes y prácticas productivas y culturales. Son viajes de pocos días de duración donde trabajan directamente con la gente, aunque en ocasiones sólo hay observación y demostración de experiencia, reforzando la formación profesional fuera del aula. 
Tabla 2. Viajes de estudio de la Licenciatura en Desarrollo Sustentable

\begin{tabular}{|c|c|c|c|c|c|c|}
\hline 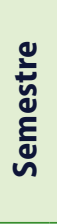 & $\begin{array}{l}\text { Asignatura de } \\
\text { vinculación } \\
\text { comunitaria } \\
\text { (duración del viaje } \\
\text { en días) }\end{array}$ & $\begin{array}{l}\text { Región o } \\
\text { zona de } \\
\text { Chiapas }\end{array}$ & $\begin{array}{c}\text { Ecosistema } \\
\text { o recurso } \\
\text { natural }\end{array}$ & $\begin{array}{l}\text { Grupos } \\
\text { étnicos }\end{array}$ & $\begin{array}{l}\text { Sistemas } \\
\text { Productivos }\end{array}$ & $\begin{array}{l}\text { Conceptos } \\
\text { integradores }\end{array}$ \\
\hline 1 & $\begin{array}{l}\text { Prácticas de } \\
\text { Vinculación con la } \\
\text { comunidad (1 día) }\end{array}$ & $\begin{array}{l}\text { Museo de } \\
\text { historia y } \\
\text { Zoológico }\end{array}$ & $\begin{array}{l}\text { Selva Baja } \\
\text { Caducifolia }\end{array}$ & Todos & Todos & $\begin{array}{l}\text { Comunidad } \\
\text { Desarrollo } \\
\text { Vinculación } \\
\end{array}$ \\
\hline 2 & $\begin{array}{l}\text { Vinculación con la } \\
\text { comunidad } \\
\text { (1 día) }\end{array}$ & $\begin{array}{l}\text { Altos y } \\
\text { Centro }\end{array}$ & $\begin{array}{l}\text { Bosque de } \\
\text { pino y selva } \\
\text { baja }\end{array}$ & $\begin{array}{l}\text { Zoque } \\
\text { Tsotsil } \\
\text { Tseltal } \\
\text { Mestizo }\end{array}$ & $\begin{array}{c}\text { Milpa } \\
\text { Caña } \\
\text { Hortaliza }\end{array}$ & $\begin{array}{c}\text { Interculturalidad } \\
\text { Ecología } \\
\text { Economía }\end{array}$ \\
\hline 3 & $\begin{array}{l}\text { El conocimiento en la } \\
\text { integración de saberes } \\
\text { ( } 3 \text { días) }\end{array}$ & Frailesca & Selva baja & $\begin{array}{l}\text { Mestizo } \\
\text { Tsotsil }\end{array}$ & $\begin{array}{l}\text { Maíz } \\
\text { Frijol }\end{array}$ & $\begin{array}{l}\text { Tierra } \\
\text { Maíz } \\
\text { Agua }\end{array}$ \\
\hline 4 & $\begin{array}{l}\text { La comprensión en el } \\
\text { diálogo de saberes ( } 3 \\
\text { días) }\end{array}$ & El Triunfo & $\begin{array}{l}\text { Bosque } \\
\text { mesófilo de } \\
\text { montaña }\end{array}$ & $\begin{array}{l}\text { Mestizo } \\
\text { Tseltal }\end{array}$ & $\begin{array}{c}\text { Café } \\
\text { Rec. Forestal } \\
\text { Ecoturismo } \\
\end{array}$ & $\begin{array}{c}\text { Bosque } \\
\text { Café } \\
\text { Comunidad }\end{array}$ \\
\hline 5 & $\begin{array}{l}\text { La generación de } \\
\text { saberes ambientales } \\
\text { y su aplicación en el } \\
\text { ámbito comunitario } \\
\text { (3 días) }\end{array}$ & Soconusco & Manglar & $\begin{array}{l}\text { Mestizo } \\
\text { Mam } \\
\text { Akateco }\end{array}$ & $\begin{array}{l}\text { Manejo del } \\
\text { café y cacao, } \\
\text { ecoturismo }\end{array}$ & $\begin{array}{l}\text { Agroecología } \\
\text { Rec. Naturales } \\
\text { Población }\end{array}$ \\
\hline 6 & $\begin{array}{l}\text { Análisis de los } \\
\text { saberes ambientales } \\
\text { generados y su } \\
\text { mediación para la } \\
\text { sustentabilidad (4 días) }\end{array}$ & $\begin{array}{l}\text { Norte } \\
\text { Zoque }\end{array}$ & $\begin{array}{l}\text { Bosque de } \\
\text { pino-encino }\end{array}$ & Zoque & $\begin{array}{l}\text { Manejo } \\
\text { forestal } \\
\text { Ecoturismo }\end{array}$ & $\begin{array}{l}\text { Educación } \\
\text { Territorio } \\
\text { Producción } \\
\text { animal }\end{array}$ \\
\hline 7 & $\begin{array}{l}\text { Síntesis de los saberes } \\
\text { ambientales en la } \\
\text { instrumentación } \\
\text { de proyectos de } \\
\text { desarrollo comunitario } \\
\text { (4 días) }\end{array}$ & $\begin{array}{c}\text { Selva } \\
\text { Lacandona }\end{array}$ & $\begin{array}{l}\text { Selva alta } \\
\text { perennifolia }\end{array}$ & $\begin{array}{l}\text { Tseltal } \\
\text { Tojolabal } \\
\text { Ch'ol }\end{array}$ & $\begin{array}{c}\text { Manejo de la } \\
\text { vida silvestre } \\
\text { Etnoecología } \\
\text { Huertos }\end{array}$ & $\begin{array}{c}\text { Etnia } \\
\text { Tecnología } \\
\text { Seguridad } \\
\text { alimentaria }\end{array}$ \\
\hline 8 & $\begin{array}{l}\text { Evaluación del } \\
\text { desarrollo sustentable } \\
\text { ( } 5 \text { días) }\end{array}$ & $\begin{array}{l}\text { Oaxaca, } \\
\text { México }\end{array}$ & $\begin{array}{l}\text { Zonas } \\
\text { Áridas }\end{array}$ & $\begin{array}{l}\text { Mestizo } \\
\text { Zapoteco } \\
\text { Mixe } \\
\text { Nahuatl }\end{array}$ & $\begin{array}{c}\text { Mezcal } \\
\text { Ecoturismo } \\
\text { Diversidad } \\
\text { cultural }\end{array}$ & $\begin{array}{c}\text { Género } \\
\text { Políticas de } \\
\text { desarrollo } \\
\text { Comunicación }\end{array}$ \\
\hline
\end{tabular}

Fuente: UNICH, 2005. 
La formación de profesionales en desarrollo sustentable

en un programa de educación superior intercultural

Joaquín Peña Piña

\subsection{El impulso a la gestión de alternativas y proyectos de desarrollo comunitario}

El proceso de vinculación bajo el enfoque del diálogo de saberes permite a los estudiantes el fortalecimiento académico para la identificación de problemas, necesidades y potencialidades de las comunidades de trabajo, a partir de un diálogo horizontal con la comunidad. Sin embargo, durante el proceso de vinculación es muy común que surjan demandas en las mismas comunidades de vinculación para la resolución de problemas y necesidades. Esta situación requiere de la atención y desarrollo de procesos organizativos o proyectos locales que implican un proceso de trabajo profesional.

Aunque la instrumentación de estos proyectos en la comunidad no forma parte de la misión del programa educativo, siempre hay equipos de estudiantes interesados en contribuir al desarrollo de sus comunidades de vinculación, pero se encuentran con la limitante de no contar con una asesoría profesional. Algunas experiencias de gestión de proyectos ante instancias gubernamentales mostraron resultados exitosos, pero los trámites burocráticos y la falta de apoyos para movilizarse y concluir la comprobación en tiempo y forma, acabaron por distraer su proceso educativo.

Esta experiencia condujo a los profesores de desarrollo sustentable a replantear como una estrategia la gestión de proyectos comunitarios, pero de manera externa al modelo educativo. La figura jurídica propuesta fue como Agencia de Desarrollo, para atender actividades de capacitación e incubación de proyectos productivos y sociales. Se propuso su coordinación al área de extensión y vinculación universitaria por su relación con actores externos e institucionales de la sociedad. La propuesta se desarrolló pero no se operó ante la falta de visión de los responsables de su instrumentación, y con ello se evitó consolidar un proceso de vinculación de una manera interactiva e integral entre el extensionismo y la vinculación académica, mediante la participación activa de los estudiantes, profesores, comunidades, instancias de financiamiento de la sociedad civil, la iniciativa privada y el gobierno (Figura 1). A pesar de ello, la experiencia fue valiosa, ya que la vinculación se reafirmó como un proceso educativo de la licenciatura que facilita el diagnóstico y el conocimiento de los problemas y necesidades de las comunidades, mediante el diálogo de saberes y el acompañamiento de estudiantes y profesores de la academia. Los problemas elegidos por los estudiantes con posibilidades de financiamiento, pasarían entonces al área de extensión y vinculación social como parte de un proceso externo, donde la Agencia de Desarrollo fungiría como la asesora del proceso de intervención en la comunidad, con la ventaja de que 
los estudiantes tienen el conocimiento cercano de la comunidad, su cultura y necesidades de los beneficiarios. De este modo, se prevé construir un círculo colaborativo entre la vinculación académica y la vinculación social universitaria para el logro del impacto social, económico y cultural.

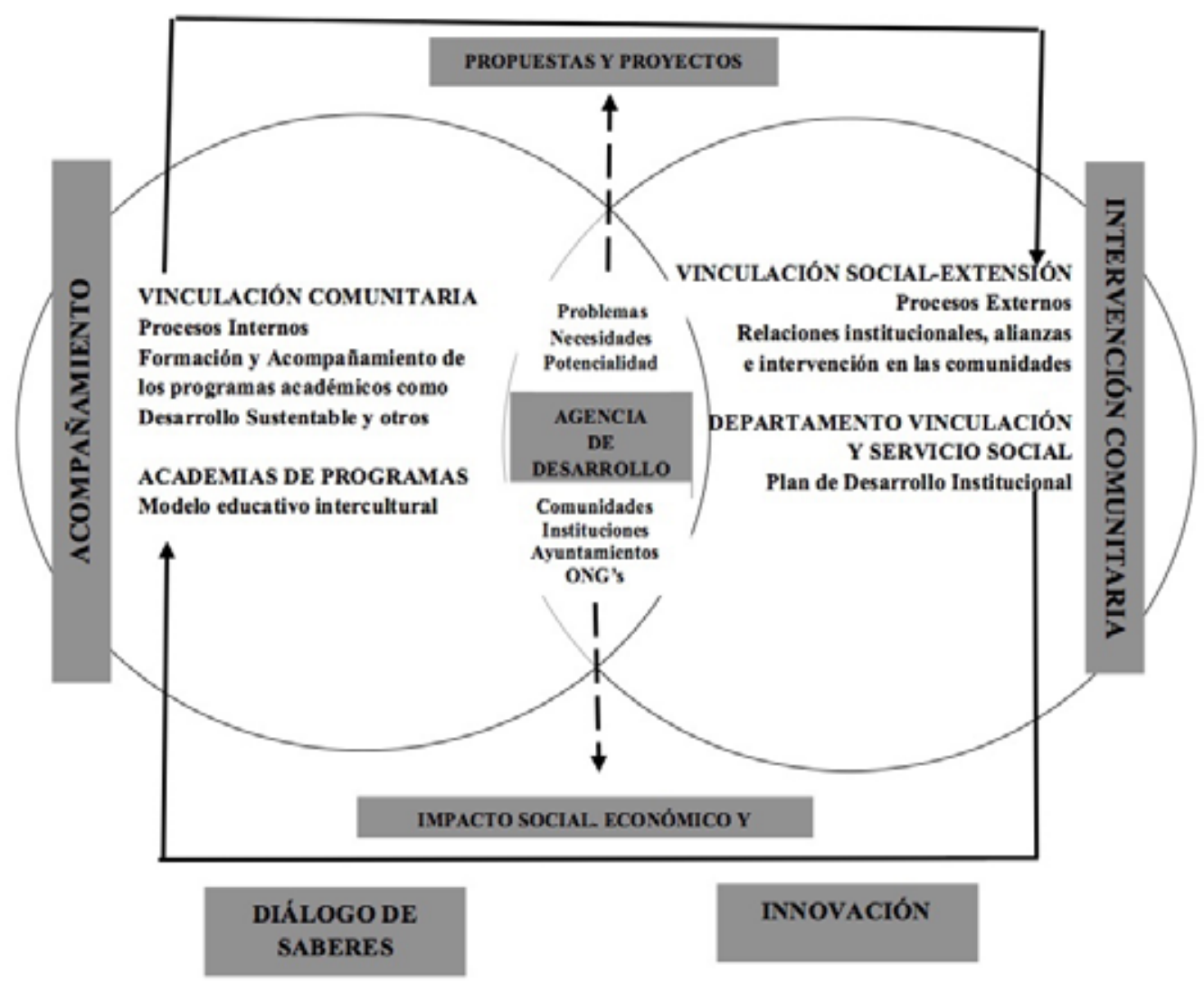

Figura 1. Integración de procesos de gestión comunitaria a partir de la vinculación y el extensionismo

Fuente: Elaboración propia con base en el trabajo en aula y en los foros de discusión.

Como parte del proceso educativo es necesaria su evaluación desde la perspectiva del diálogo de saberes, pero no sólo en su práctica áulica sino en todos los aspectos mencionados anteriormente. Como se puede observar, la evaluación del proceso de 
vinculación comunitaria se enfrenta al reto de la complejidad de la formación académica del estudiante y a la necesidad de integrar los saberes y prácticas locales en la comprensión y logro de la sustentabilidad y la interculturalidad.

En la práctica áulica, se aplicó un procedimiento sencillo y ágil para contar con datos inmediatos útiles al profesor y a los mismos estudiantes sobre los conceptos integradores y los conocimientos y prácticas de las asignaturas del semestre. Para identificar la situación de los ejes integradores, al inicio del curso de vinculación se aplicó la técnica SQA (lo que Sé, lo que Quiero aprender y lo que Aprendí), para indagar entre los estudiantes sus conocimientos y expectativas de aprendizaje (Pimienta, 2012). La información asentada en un formato permitió contar en unos minutos con el diagnóstico general para una mejor planeación didáctica y atender las necesidades del estudiante. Para evaluar el proceso de aprendizaje de las asignaturas se utilizó un formato-matriz con las siguientes columnas: Asignatura, Saber Conceptual (¿Cuáles son los conceptos clave vistos en el curso), Saber Hacer ( $i$ Cómo aplico ese conocimiento?), Saber Ser (¿Con qué actitud reflexiono y actúo?), Saber Saber (iCómo lo aprendí?) y otras tres columnas con los conceptos integradores del semestre (¿Cómo relaciono el aprendizaje obtenido en la asignatura con cada eje integrador?), elaborado de manera individual por los estudiantes y entregado mensualmente. Después, se socializaba colectivamente en un taller integrador cuya discusión resultaba en un aprendizaje colectivo y permitía la reflexión continua del proceso de aprendizaje.

Para evaluar el trabajo comunitario el análisis de la sustentabilidad es fundamental y es un elemento central de su formación profesional. En el caso del desarrollo sustentable, se manejan los términos de Bienestar, Buen Vivir o la Calidad de vida, destacando en ellas esta última (Contreras \& Cordero, 1994). Con una formación académica basada en el diálogo de saberes, en la evaluación se puede identificar la experiencia teórico-práctica adquirida y el desarrollo de criterios y alternativas a partir de los mismos estudiantes y el acompañamiento de los profesores y los actores locales.

\section{Las lecciones aprendidas}

La experiencia aporta elementos y procedimientos académicos que se fueron desarrollando a partir de la práctica de la vinculación comunitaria en las primeras generaciones del modelo educativo intercultural en la UNICH. Su operación y resultados

pretenden contribuir al mejoramiento de la práctica docente y al logro educativo y 
formativo de los estudiantes en desarrollo sustentable, así como al conocimiento y revaloración de la realidad en las comunidades. Como paradigma, el desarrollo sustentable es un concepto complejo, como lo es el mismo proceso de vinculación con las comunidades. La experiencia indica que en el contexto chiapaneco las comunidades cuentan con una vasta riqueza biológica y cultural, manifiesta en el conocimiento profundo de su entorno, en los saberes y las prácticas locales y en la importancia de la vida comunitaria como espacio de aprendizaje para los estudiantes y profesores.

Así, el análisis del diálogo de saberes es fundamental en el proceso formativo universitario, ya que demuestra la extensa riqueza de saberes y prácticas que poseen las comunidades, a la par de los saberes disciplinarios y académicos del aula para ubicarlos al mismo nivel de importancia y formación, enriquecidos a su vez por los saberes y experiencia del estudiante. González (2011) presenta su experiencia con la Universidad Intercultural del Estado de México mediante el trabajo en una comunidad indígena, donde también hay un intercambio recíproco de saberes entre la universidad y la sociedad, corroborando la importancia de la vinculación en el modelo educativo intercultural.

Para ello, es necesario el uso de diversos recursos y estrategias didácticas y de discusión, lo que implica un trabajo no sólo del estudiante sino de los profesores y del entorno académico en su dinámica universitaria.

La información generada pretende contribuir a una reflexión permanente sobre el proceso de vinculación en las universidades interculturales, cuyo modelo representa una alternativa viable entre los jóvenes para el logro de una educación superior acorde a su contexto. La experiencia aporta elementos del proceso de vinculación para dirigir esfuerzos en el futuro hacia una mayor construcción teórica (Campos \& Sánchez, 2005), que abone a la comprensión y fortalecimiento de un modelo educativo con un gran potencial para promover la interculturalidad en la educación superior, especialmente en el estado de Chiapas por su diversidad biocultural.

El diálogo de saberes fortalece la profesionalización del estudiante durante el mismo proceso formativo universitario, pues al egresar cuentan con la experiencia, visión y actitud suficientes para promover los procesos de sustentabilidad e interculturalidad en las comunidades. Los foros de egresados en las primeras generaciones dan cuenta de las fortalezas y debilidades del proceso educativo en la UNICH, así como la importancia y aportes que tuvo el diálogo de saberes en la formación profesional (UNICH, 2011). 
El análisis del eje de vinculación constata la riqueza innata de los saberes que poseen las comunidades, pero también da cuenta de la acelerada fragmentación que sufren en lo económico, social y cultural, manifiesta en la pérdida de la colectividad o la participación social, entre otros aspectos, como consecuencia de las políticas y embate del modelo de desarrollo actual, que amenaza la riqueza cultural y la cohesión comunitaria. Por ello, mediante la vinculación se advierte que la vida comunitaria tiene su propio dinamismo regido por normas socioculturales donde las relaciones sociales y el diálogo son intensos, y se evidencian en las asambleas, la participación social, los valores comunitarios, el trabajo colectivo, las fiestas tradicionales o los proyectos colectivos, entre otros aspectos.

El avance en la concepción del diálogo de saberes como proceso formativo, nos permite ubicar que éste sólo puede lograrse de manera multifuncional y articulada, en tanto demanda la participación de estudiantes, profesores y autoridades administrativas en el proceso educativo, si lo que se pretende lograr es la formación de profesionales con una nueva visión de la gestión comunitaria, y un mayor vínculo de la universidad con la sociedad.

Para finalizar, la formación profesional a partir del proceso de vinculación universitario y el diálogo de saberes permite a los nuevos profesionales en desarrollo sustentable estar en mejores condiciones para promover una nueva manera de reapropiación social de la naturaleza, un cambio en la sustentabilidad a partir del diálogo con los actores sociales, y la generación de alternativas de desarrollo en equilibrio con la naturaleza, como principios esenciales de la formación de profesionales en desarrollo sustentable.

\section{Lista de referencias}

Brundtland, G. H. (1987). Report of the World Commission on Environment and Development: Our common future (Reporte A/42/427). Recuperado de http://www. un-documents.net/wced-ocf.htm

Campos, G., \& Sánchez, G. (2005). La vinculación universitaria: ese oscuro objeto del deseo. Revista Electrónica de Investigación Educativa, 7(2), 1-13. Recuperado de http://redie.uabc.mx/redie/article/view/134

Casillas, L., \& Santini, L. (2006). Modelo intercultural. Modelo educativo. México: Coordinación General de Educación Intercultural y Bilingüe-Secretaría de Educación Pública. 
Conteras, H., \& Cordero, A. (1994). Ambiente, desarrollo sustentable y calidad de vida. Caracas: Universidad Central de Venezuela.

Echeverría, E. (2016). El papel de la comunidad de diálogo filosófico en la formación de la identidad del adolescente [Página Web]. Recuperado de http://www.celafin. org/adolescente.html\#inicio

Freire, P. (2007). ¿Extensión o comunicación? La concientización en el medio rural. México: Siglo Veintiuno.

Gómez, J. L. (enero-junio, 2014). Del desarrollo sostenible a la sustentabilidad ambiental. Revista de la Facultad de Ciencias Económicas: Investigación y Reflexión, 22(1), 115-136.

González, F. (septiembre-diciembre, 2011). La vinculación universitaria en el modelo de educación superior intercultural en México. La experiencia de un proyecto. Ra Ximhai, $7(3)$, 381-394.

Leff, E. (2004). Racionalidad ambiental y diálogo de saberes: significancia y sentido en la construcción de un futuro sustentable. Polis. Revista Latinoamericana, 2(7), 28. Recuperado de https://polis.revues.org/6232

Navarrete, F. (2008). Los Pueblos Indígenas de México. México: Comisión Nacional para el Desarrollo de los Pueblos Indígenas.

Pérez, M. L. (2005). La comunidad indígena contemporánea. Límites, fronteras y relaciones interétnicas. En M. Lisbona (Coord.), La comunidad a debate. Reflexiones sobre el concepto de comunidad en el México contemporáneo (pp. 87-10o). México: Colegio de Michoacán/Universidad de Ciencias y Artes de Chiapas.

Pérez, M. L., \& Argueta, A. (marzo, 2011). Saberes indígenas y diálogo intercultural. Cultura y Representaciones Sociales, 5(10), 31-56. Recuperado de http://www. journals.unam.mx/index.php/crs/article/view/24448

Pimienta, J. (2012). Estrategias de enseñanza-aprendizaje. Docencia universitaria basada en competencias. México: Pearson.

Universidad Intercultural de Chiapas. (2005). Programa académico de la Licenciatura en Desarrollo Sustentable. San Cristóbal de Las Casas, Chiapas: Academia de Desarrollo Sustentable-Universidad Intercultural de Chiapas.

Universidad Intercultural de Chiapas. (2008). Programa académico de la Licenciatura en Desarrollo Sustentable. Actualización. San Cristóbal de Las Casas, Chiapas: Academia de Desarrollo Sustentable-Universidad Intercultural de Chiapas.

Universidad Intercultural de Chiapas. (2011). Primer foro de Intercambio de Experien- 
cias Laborales de los Egresados de la Primera Generación de la UNICH. San Cristóbal de Las Casas, Chiapas: Autor.

Walsh, C. (2002). (De)construir la interculturalidad. Consideraciones críticas desde la política, la colonialidad y los movimientos indígenas y negros en el Ecuador. En N. Fuller (Ed.), Interculturalidad y política: desafíos y posibilidades (pp. 115142). Lima: Red para el desarrollo de las Ciencias Sociales en el Perú/Benemérita Universidad Católica del Perú. 